\title{
Dentin Tubule Occlusion Potential of Novel Dentifrices Having Fluoride Containing Bioactive Glass and Zinc Oxide Nanoparticles
}

\author{
Abdul Samad Khan ${ }^{a}$ Imran Farooq $^{b}$ Kawther Moosa Alakrawi ${ }^{c}$ Hina Khalid $^{d}$ \\ Omar Waqas Saadi ${ }^{e}$ Abbas Saeed Hakeem ${ }^{f}$
}

a Department of Restorative Dental Sciences, College of Dentistry, Imam Abdulrahman Bin Faisal University, Dammam, Saudi Arabia; ${ }^{b}$ Department of Biomedical Dental Sciences, College of Dentistry, Imam Abdulrahman Bin Faisal University, Dammam, Saudi Arabia; ' College of Dentistry, Imam Abdulrahman Bin Faisal University, Dammam, Saudi Arabia; ${ }^{d}$ Interdisciplinary Research Centre in Biomedical Materials, COMSATS University Islamabad, Lahore Campus, Lahore, Pakistan; 'Department of Mechanical Engineering, Khalifa University, Abu Dhabi, United Arab Emirates; ${ }^{\mathrm{f} C e n t e r}$ of Excellence in Nanotechnology, King Fahd University of Petroleum and Minerals, Dhahran, Saudi Arabia

\section{Highlights}

- We analyzed the potential of dentin tubule occlusion of two novel experimental dentifrices, having fluoride containing bioactive glass and zinc oxide nanoparticles.

- Experimental dentifrices were found capable of occluding more tubules than fluoride dentifrice, and their results are comparable to commercially available bioactive glass dentifrices.

- The results can aid in offering appropriate recommendations for dentifrice for patients, especially those suffering from dentin sensitivity.

\section{Keywords}

Bioactive glass · Fluoride · Zinc · Dentin sensitivity · Scanning electron microscopy

\begin{abstract}
Objective: To compare the in vitro potential of dentin tubule occlusion of two novel experimental dentifrices consisting of fluoride containing bioactive glass (BG) with zinc oxide nanoparticles. Materials and Methods: Forty-eight dentin discs $(n=48)$ were divided into 6 groups $(n=8)$, based on their brushing dentifrices: Group $1=$ artificial saliva (AS; control); Group 2 = fluoride dentifrice (Colgate Palmolive ${ }^{\circledR}$, UK); Group 3 = experimental nonactive agent dentifrice; Group $4=$ experimental dentifrice with 1.5\% BG; Group $5=$ experi-
\end{abstract}

\section{KARGER}

karger@karger.com www.karger.com/mpp (c) 2019 The Author(s) Published by S. Karger AG, Basel

Karger

Open access

This is an Open Access article licensed under the Creative Commons Attribution-NonCommercial-4.0 International License (CC BY-NC) (http://www.karger.com/Services/OpenAccessLicense), applicable to the online version of the article only. Usage and distribution for commercial purposes requires written permission. mental dentifrice with 4\% BG; and Group $6=$ BioMinF $^{\odot}$ dentifrice. Postbrushing, the discs were subjected to acidic challenge with $6 \%$ wt citric acid $(\mathrm{pH}=4.0$ ) for $1 \mathrm{~min}$. Scanning electron microscope (SEM) and energy-dispersive $\mathrm{X}$-ray (EDX) spectroscopy were performed pre-and post-citric acid challenges, and percentages of tubule occlusion assessed. Results: SEM micrographs of group 1 (AS) show no tubule occlusion ( $0 \%$ ), whereas those of groups 2 and 3 show partial tubule occlusion ( 25 to $<50 \%$ of tubules occluded). The SEM micrographs of dentifrices containing fluoride-BG (groups 4 , 5 , and 6 ) show that most of the tubules ( $>50$ and $<100 \%$ ) were occluded. For all the groups (excluding group 1), preand post-citric acid challenge values are statistically significant $(p<0.05)$. EDX analysis reveals the presence of zinc in experimental dentifrices only. Conclusion: The results of 
novel experimental dentifrices are comparable to those of the BioMinF ${ }^{\odot}$, in terms of tubule occlusion. Dentifrices containing BG could serve as an alternative in dentin sensitivity management.

(c) 2019 The Author(s)

Published by S. Karger AG, Basel

\section{Introduction}

Dentin is a mineral-bearing tissue, composed of tiny dentinal tubules, whose exposure due to enamel loss and gingival recession results in dentin hypersensitivity $(\mathrm{DH})$ [1]. $\mathrm{DH}$ is a common problem, and its prevalence has been reported to be as high as $74 \%$ [1]. The most widely accepted theory is the hydrodynamic theory, which explains that $\mathrm{DH}$ is caused by the exposure of dentinal tubules in which the movement of fluids, in response to stimuli, activates nerve endings in the pulp and causes sharp pain for a short duration [2]. The extrinsic factors that contribute to dental erosion leading to the exposure of tubules are related to acids of dietary or medicinal origin; besides, they are also associated with certain behavioral factors. The causes of $\mathrm{DH}$ include caries, gingival recession, tooth wear, and fractures [3].

One solution to minimize DH is to block the open dentinal tubules [1]. With aging, dentin can change to sclerotic dentin, as the tubules can get occluded by minerals [4]. However, this process is slow, and the patient may stay in pain for a long time. It has been reported earlier that the use of desensitizing dentifrices could reduce $\mathrm{DH}$ immediately and effectively [5]. It is also possible to reverse demineralization by deploying mineralizing agents on the tooth surface. Bioactive glass (BG) is one such type of mineralizing agent, which is capable of bonding chemically to the hard dental tissues [6]. The components of BG are oxides of calcium, sodium, phosphorus, and silica, in ratios that impart bioactivity [7]. These BGs were introduced in the late 1960s primarily for osteogenesis; however, since then, their inclusion in various dental products, especially dentifrices, has been increasing [8]. As the bone and dentin are similar in structure, the use of BGs on dentin could prove useful in attaining clinical benefits [9].

The reaction of BG in aqueous solution brings about a change in its structure and chemical composition, as it dissolves and leads to the formation of hydroxycarbonated apatite (HAP) [8]. The fluoride of traditional BGs is not inside their glass structure, although that would be more beneficial in ensuring the formation of strong fluorapatite (FAP) crystals [10]. One such commercially available toothpaste is BioMin $^{\complement}$, which has shown good tubule occlusion properties in a previous in vitro study [11]. Based on a recent in vitro study, Kanwal et al. [12] conclude that incorporation of fluoride in a BG toothpaste helps it in forming fluoridated apatite in Tris buffer solution, which has better clinical durability. In addition, fluoride in combination with other desensitizing agents in toothpaste shows increased efficacy in caries prevention and desensitization [13].

This study was conducted out to develop BGs of new compositions and test them for their dentin tubule occlusion capability. Accordingly, two novel compositions of dentifrices, having fluoride-containing $\mathrm{BG}$ and nanozinc oxide $(\mathrm{ZnO})$ powders as active ingredients, were evaluated for their potential in dentin tubule occlusion and the results compared with that of a standard fluoride dentifrice and BioMinF ${ }^{\odot}$. The null hypothesis $\left(\mathrm{H}_{0}\right)$ was that all tested dentifrices will have similar dentin tubule occlusion competence.

\section{Materials and Methods}

Ethical clearance was obtained from the Scientific Research Unit of the institute, and all the ethical protocols were strictly followed.

\section{Composition of Dentifrices}

A standard fluoride dentifrice (Colgate-Palmolive $\left.{ }^{\circledR}, \mathrm{UK}\right)$ and BioMinF $^{\complement}$ were used in this study for the purpose of comparison with the new experimental dentifrices. A nonactive agent of dentifrice composition and 2 experimental dentifrice compositions with active agents were developed and used in this study. All the chemicals used were of analytical grade. Initially, the basic ingredients were mixed in optimum ratio and allowed to form a homogeneous solution. Then $1.5 \%$ wt. of titanium dioxide (Sigma Aldrich, St. Louis, MO, USA) and 3\% wt. of $\mathrm{ZnO}$ nanoparticles [14] were added in increments to avoid agglomeration. Fluoride-BG $(7.5 \% \mathrm{~mol})$ was synthesized following the sol-gel precipitation method of Gul et al. [15]. The fluoride-BG thus obtained was incorporated into the experimental toothpastes in different concentrations, that is, 1.5 and $4 \% \mathrm{wt} / \mathrm{wt}$. The composition of each dentifrice (with its active and basic ingredients) is given in Table 1.

Preparation of Dentin Discs and Grouping of Specimens

For this study, extracted noncarious teeth were collected from Oral and Maxillofacial Surgery Clinics of the institute. Forty-eight dentin discs of $2.0 \mathrm{~mm}( \pm 0.2 \mathrm{~mm})$ size were prepared by cutting each tooth horizontally (mesio-distally) over cemento-enamel junction, utilizing cooled diamond saw (Isomet $^{\circledR} 5000$ Linear Precision Saw, Buehler Ltd., Lake Bluff, IL, USA). The occlusal enamel, $2.5 \mathrm{~mm}$ down the cusp tip, was also removed and the teeth were cut mesio-distally. The upper surfaces of the dentin discs were marked, and the unmarked surfaces were etched with $37 \%$ orthophosphoric acid for $20 \mathrm{~s}$ to open the tubules and to free them from any organic material. Post-etching, the discs were washed with dis- 
Table 1. Showing composition of all the dentifrices used in this study

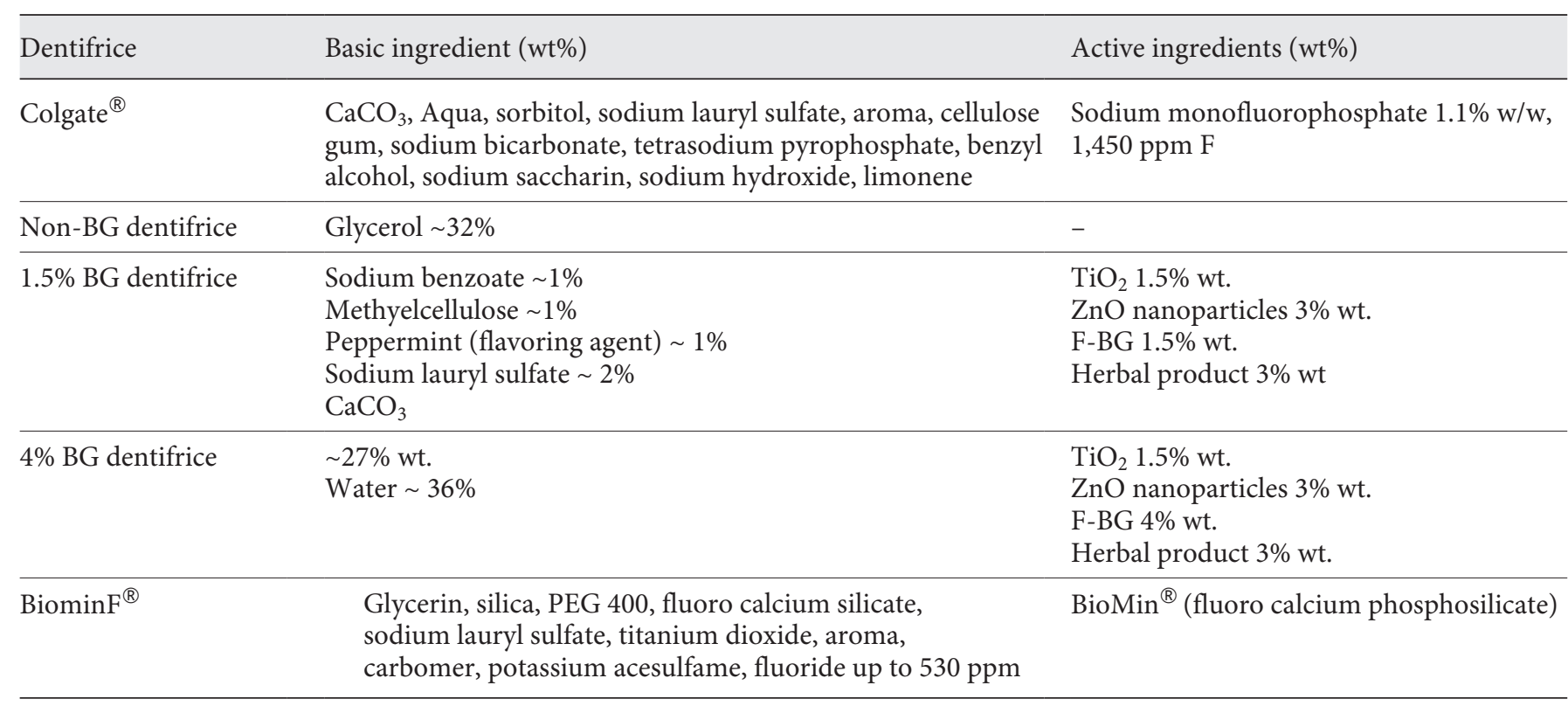

BG, bioactive glass; $\mathrm{CaCO}_{3}$, calcium carbonate ; $\mathrm{TiO}_{2}$, titanium dioxide; $\mathrm{ZnO}$, zinc oxide; F-BG, fluoride-bioactive glass;

tilled water for $1 \mathrm{~min}$ and randomly divided into the following 6 groups, each group receiving 8 discs $(n=8)$, based on their treatment with different dentifrices: Group 1 = discs brushed with artificial saliva (AS; control); Group 2 = discs brushed with fluoride dentifrice (Colgate Palmolive ${ }^{\circledR}$, UK); Group 3 = discs treated with experimental non-active agent dentifrice; Group $4=$ discs treated with experimental dentifrice, having 1.5\% BG; Group 5 = discs brushed with experimental dentifrice, containing 4\% BG; and Group 6 = discs treated with Biomin-F dentifrice.

The AS was prepared by mixing the following chemicals in 1,000 $\mathrm{mL}$ of deionized water, as proposed by Fusayama et al. [16]: $\mathrm{NaCl}: 0.400$ g, KCl: 0.400 g, $\mathrm{NaH}_{2} \mathrm{PO}_{4} . \mathrm{H}_{2} \mathrm{O}: 0.69$ g; $\mathrm{CaCl}_{2} . \mathrm{H}_{2} \mathrm{O}$ : $0.795 \mathrm{~g} ; \mathrm{Na}_{2} \mathrm{~S} .9 \mathrm{H}_{2} \mathrm{O}: 0.005 \mathrm{~g}$. The $\mathrm{pH}$ of the freshly prepared AS was 5.5 , which was adjusted to the neutral $\mathrm{pH}$ of 7.0 by adding aliquots of $\mathrm{NaOH}[10]$.

\section{Tooth Brushing Protocol for Dentin Discs}

Discs of group 1 were brushed with $1 \mathrm{~mL}$ AS and those of other groups with $1 \mathrm{~g}$ of their respective dentifrices for $2 \mathrm{~min}$, twice a day (once in the morning and once in the afternoon) for 7 days. The dentifrice was mixed with deionized water in the ratio of $1: 2$, and a load of $200 \mathrm{~g}$ was applied to the toothbrush (Medium bristled, Oral $B^{\circledR}$, Pro-Flex ${ }^{\mathrm{TM}}$, USA) head to ensure that it contacted the samples. Each sample was treated for 150 cycles/min inside a toothbrush simulation machine (Toothbrush simulator; model ZM-3.8, Germany). Each day, post-brushing, the discs were washed with distilled water for $1 \mathrm{~min}$ and then returned to their respective containers containing AS at $37^{\circ} \mathrm{C}$.

\section{Citric Acid Challenge}

Post-brushing, the discs were subjected to citric acid (6\% wt.) challenge for $1 \mathrm{~min}$ under dynamic conditions. The citric acid was freshly prepared by adding $6 \mathrm{~g}$ citric acid powder (FunFresh Foods ${ }^{\circledR}$, San Clemente, CA, USA) to $100 \mathrm{~mL}$ of deionized water, and the $\mathrm{pH}$ was maintained at 4 . After citric acid exposure, the discs were washed with distilled water and left to air dry for $24 \mathrm{~h}$ before analyzing them by scanning electron microscope (SEM).

\section{Analysis of Tubule Occlusion by SEM Energy-Dispersive X-Ray} Analysis

The dentin discs were mounted on stubs, given sputter coating with gold and then analyzed, using an SEM (FEI, Inspect F50, The Netherlands) with energy-dispersive spectroscopy (EDS, Oxford Instruments, UK). The discs were evaluated pre- and post-citric acid challenges. Micrographs were taken from the central areas marked on the discs, utilizing 5 and $20 \mathrm{kV}$ electron modes, at 10 and $15 \mathrm{Kx}$ magnifications. The percentage of the blocked dentinal tubules was calculated by dividing the number of occluded tubules in a single micrograph with the total number of tubules present in that image, and then multiplying it with 100 . Three independent blinded reviewers (who were not part of the study group) were requested to analyze the percentages of tubule occlusion, following the criteria given by Pathan et al. [17]: No occlusion $=0 \%$, mostly unoccluded ( $<25 \%$ of tubules occluded), partially occluded ( 25 to $<50 \%$ of tubules occluded), mostly occluded (50 to $<100 \%$ of tubules occluded), and occluded (100\% of tubules occluded). The reviewers performed the analysis three times and then submitted the mean of their analytical results. The data of the three reviewers were pooled to arrive at the final occlusion percentage. The interreviewer agreement between the three reviewers' results, determined by using Kappa statistics, was found to be high (Kappa index: $89.9 \%$ ). 
Fig. 1. SEM micrograph of a dentin disc that was brushed with fluoride dentifrice (a) pre-citric acid challenge and (b) postcitric acid challenge.

Fig. 2. SEM micrograph of a dentin disc that was brushed with non-BG experimental dentifrice (a) pre-citric acid challenge and (b) post-citric acid challenge.
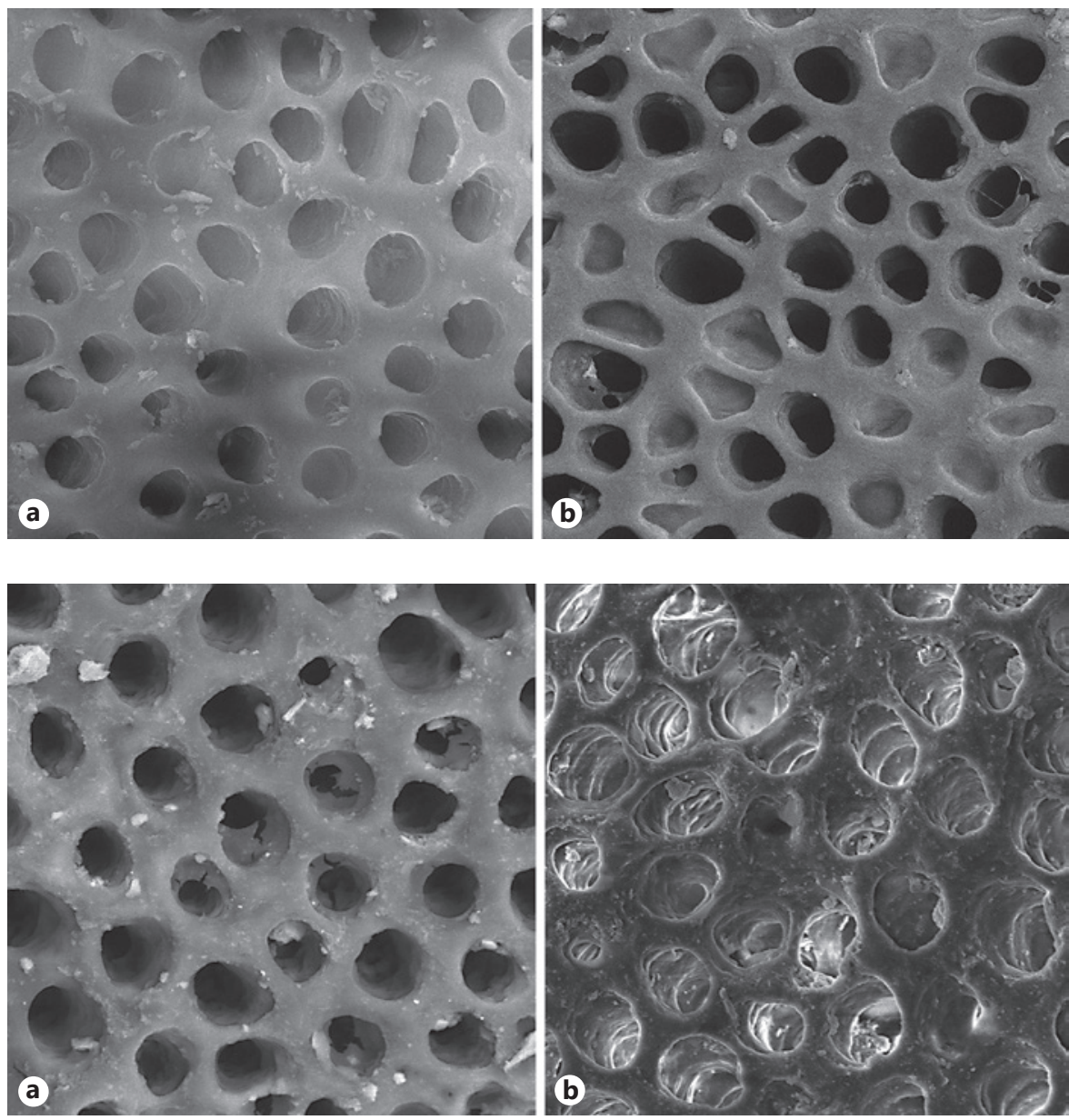

\section{Statistical Analysis}

Data analysis was performed using SPSS-20.0 (IBM product, Chicago, IL, USA). Paired sample $t$ test was applied to compare pre- and post-citric acid challenge tubule occlusion values, within each group. Tukey's HSD post hoc test was used for comparison of post-citric acid challenge results among the groups. $p$ value $\leq 0.05$ was considered a statistically significant difference between means.

\section{Results}

The SEM micrographs, belonging to group 1 (AS), showed no tubule occlusion ( $0 \%)$, both pre-and post-citric acid challenges (not shown). The SEM micrographs of group 2 (Colgate $^{\complement}$ ) showed only partial tubule occlusion ( 25 to $<50 \%$ of tubules occluded), pre- (Fig. 1a) and postcitric acid challenges (Fig. 1b). A similar finding was observed in group 3 (non-BG experimental dentifrice), where again the tubules were partially occluded (25 to $<50 \%$ of tubules occluded), pre-and post-citric acid chal- lenges (Fig. 2a, b). Compared with groups 1, 2, and 3, group 4 (containing $1.5 \% \mathrm{BG}$ ) showed superior tubule occlusion, pre- and post-citric acid challenges, and most of the tubules ( $>50$ and $<100 \%$ ) remained occluded (Fig. 3a, b). The findings for group 5 (containing 4\% BG), where most of the tubules $(>50$ and $<100 \%$ ) remained occluded, pre- and post-citric acid challenges (Fig. 4a, b), are similar to those of group 4. The findings for Group 6 are similar to those for groups 4 and 5, where again most of the tubules ( $>50$ and $<100 \%$ ) were blocked both preand post-citric acid challenges (Fig. 5a, b).

Energy-dispersive X-ray results (Table 2) show elemental composition of dentin surface post-citric acid challenge. The experimental dentifrices show that $\mathrm{Ca} / \mathrm{P}$ is approximately 2.0, whereas fluoride is rare in all the groups. It is noteworthy that the percentage of zinc is higher in the experimental dentifrices (i.e., 1.5 and $4 \%$ $\mathrm{BG}$ ) than in other dentifrice groups. Similarly, Ti (due to $\mathrm{TiO}_{2}$ ) was also visible in experimental dentifrices (Fig. 6ad). For all the groups (excluding group 1), paired sample 
Fig. 3. SEM micrograph of a dentin disc that was brushed with experimental dentifrice containing $1.5 \% \mathrm{BG}$ (a) pre-citric acid challenge and (b) post-citric acid challenge.

Fig. 4. SEM micrograph of a dentin disc that was brushed with experimental dentifrice containing 4\% BG (a) pre-citric acid challenge and (b) post-citric acid challenge.

Fig. 5. SEM micrograph of a dentin disc that was brushed with $\operatorname{BioMinF}^{\complement}$ (a) precitric acid challenge and (b) post-citric acid challenge.
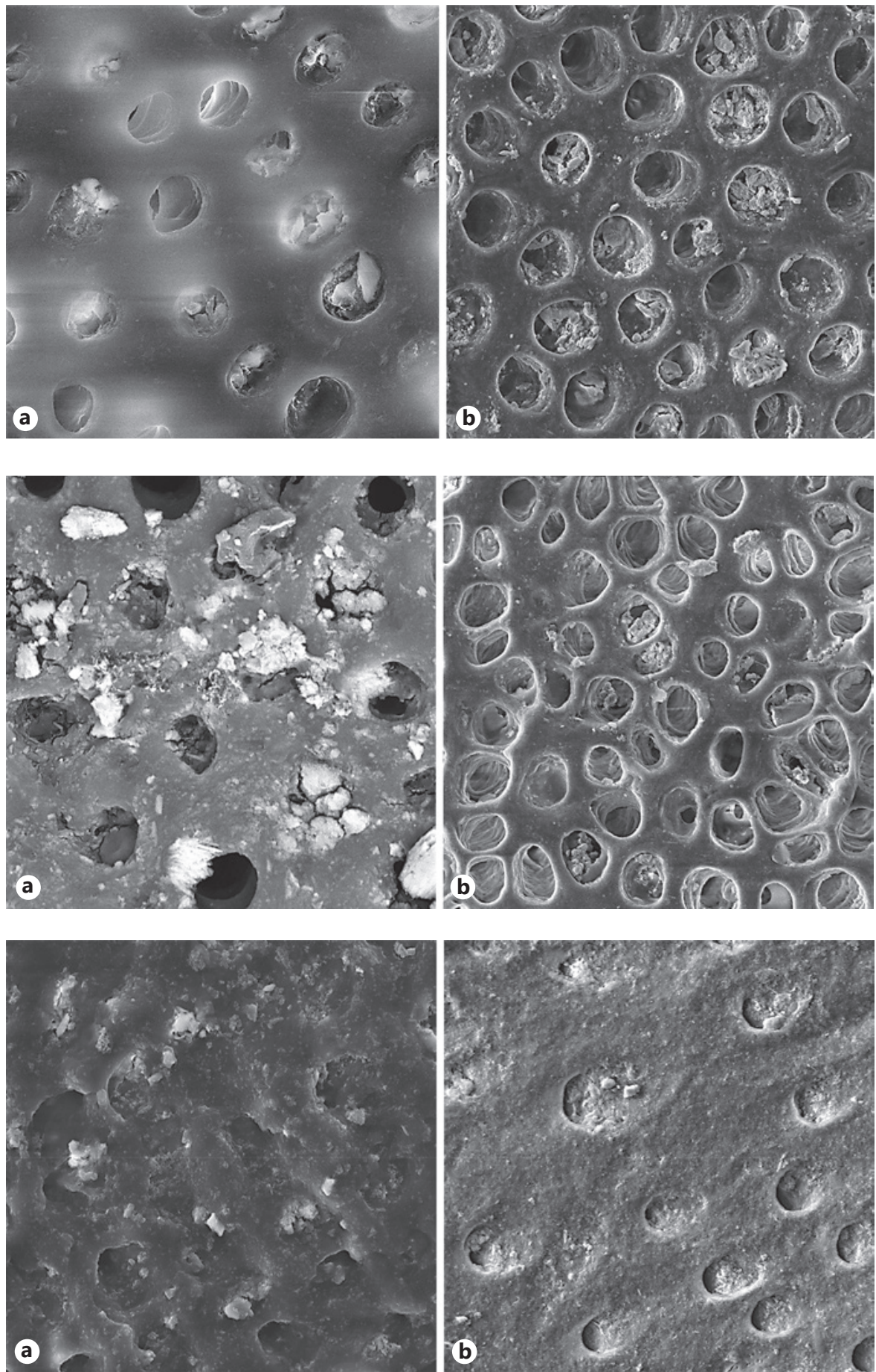

$t$ test reveals statistically significant differences $(p<0.05)$, within the group, between pre- and post-citric acid challenge values (Table 3 ). Intergroup comparisons reveal statistically significant differences $(p<0.05)$ in post-citric acid challenge tubule occlusion values (except between groups 2 and 3, groups 4-6, and groups 5 and 6; Table 4).

\section{Discussion}

This study investigated the tubule occlusion competence of two novel fluoridated-BG experimental dentifrices and compared their competences with those of standard fluoride, nonactive BG dentifrice and BioMinF ${ }^{\complement}$. 


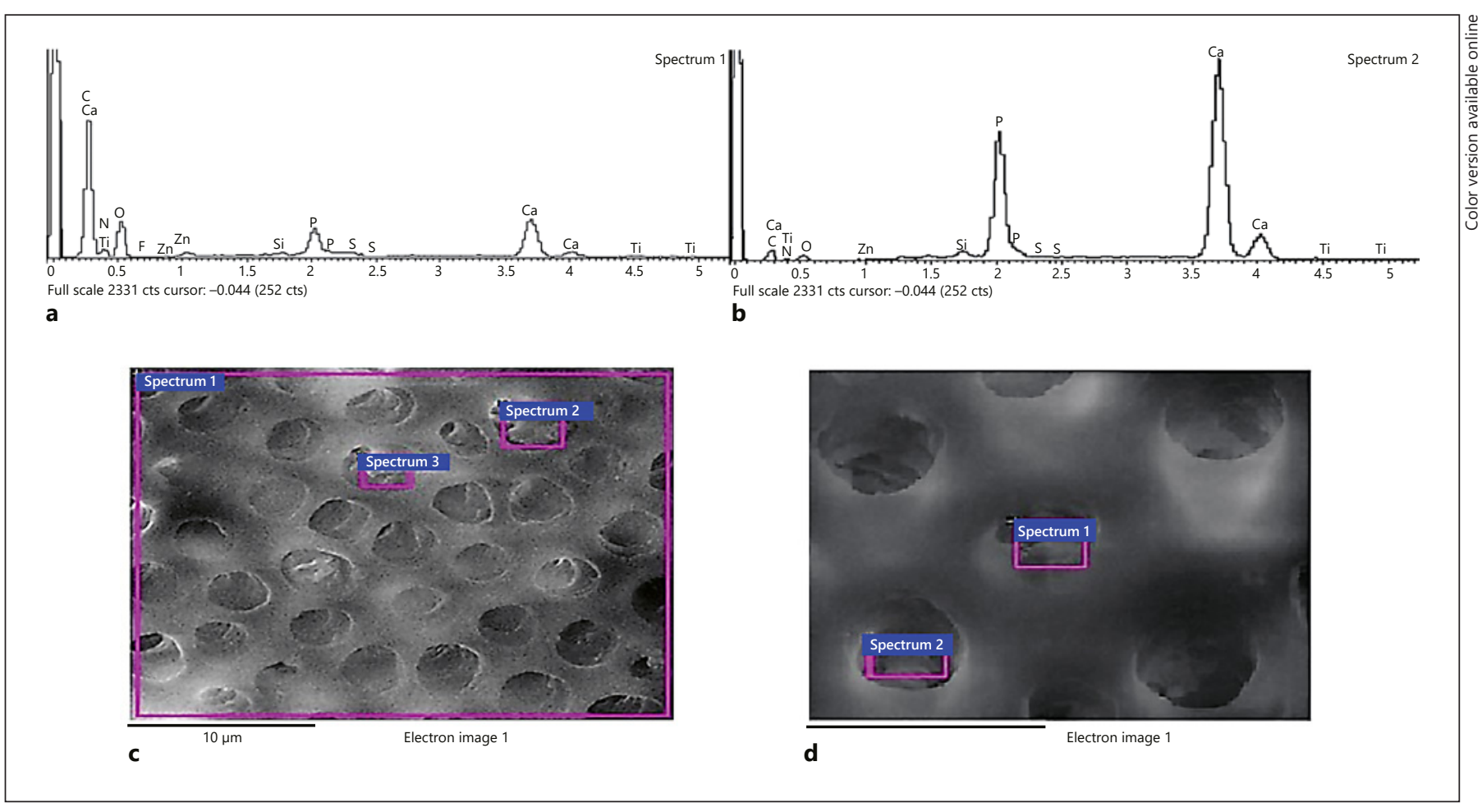

Fig. 6. Showing EDX spectra of (a) dentin discs brushed with 1.5\% BG dentifrice post citric acid challenge, (b) dentin discs brushed with 4\% BG dentifrice post citric acid challenge, (c) SEM micrograph showing EDX analysis area of dentin disc which was brushed with 1.5\% BG, and (d) SEM micrograph showing EDX analysis area of dentin disc which was brushed with $4 \%$ BG.

Table 2. EDX results showing elemental composition of different toothpaste groups

\begin{tabular}{|c|c|c|c|c|c|c|c|c|c|c|c|}
\hline \multirow[t]{2}{*}{ Groups } & \multicolumn{11}{|l|}{$\mathrm{Wt} \%$} \\
\hline & $\mathrm{C}$ & $\mathrm{N}$ & $\mathrm{O}$ & $\mathrm{F}$ & $\mathrm{Si}$ & $\mathrm{P}$ & $\mathrm{S}$ & $\mathrm{Ca}$ & $\mathrm{Ti}$ & $\mathrm{Zn}$ & total \\
\hline Group 2 & 46.60 & 21.83 & 32.15 & -1.06 & -0.03 & -0.06 & 0.06 & 0.35 & -0.01 & 0.00 & 100 \\
\hline Group 3 & 45.80 & 20.62 & 31.90 & 0.09 & 0.12 & 0.44 & 0.17 & 0.90 & -0.01 & 0.03 & 100 \\
\hline Group 4 & 38.78 & -0.47 & 39.25 & -0.13 & 0.12 & 1.37 & 0.08 & 2.63 & 17.41 & 0.94 & 100 \\
\hline Group 5 & 17.46 & 9.88 & 13.09 & -0.11 & 0.66 & 18.08 & -0.01 & 39.97 & 0.04 & 0.94 & 100 \\
\hline Group 6 & 13.63 & 0.85 & 41.55 & 0.01 & 15.18 & 0.04 & 0.49 & 30.12 & -0.05 & -0.18 & 100 \\
\hline
\end{tabular}

EDX, energy-dispersive X-ray.

The results indicate that pre- and post-citric acid challenge BioMinF ${ }^{\odot}$ dentifrices, containing $1.5 \%$ BG, and $4 \%$ $\mathrm{wt} / \mathrm{wt}$ BG were able to block most of the tubules ( $>50$ and $<100 \%)$. For the control group containing AS, no tubule occlusion was seen. Human saliva plays an important role in transporting essential ions such as calcium and phosphate to the dentin surface; however, this process by itself cannot plug these ions inside the tubules to the extent necessary to occlude them and thus decrease DH [11]. For dentifrice groups containing fluoride, and those containing no $B G$, tubule occlusion was only partial $(<25$ and $<50 \%$ ). Incorporation of fluoride in dentifrices is beneficial to tooth structure because of the strong association between the use of fluoride and prevention of dental caries [18]. Fluoride helps in the formation of FAP from HAP through substitution of hydroxyl ion for fluoride 
ion [19] in the apatite structure of enamel. The FAP is known to have better chemical stability and lesser bioresorption rate than HAP [20]. Fluoride is quite effective in enamel remineralization, but its effect on $\mathrm{DH}$ is rather limited [21]. This finding can be attributed to the fact that fluoride from a regular dentifrice can be washed away quickly by salivary flow, and the amount of FAP that can thus form is debatable [22].

Calcium and phosphate ions are integral parts of hard tissues of the human body, such as bones and teeth [23], and all the BG compositions that are available till date contain these two important ions [7]. The surface reaction that occurs when the BG comes in contact with a bone comprises the following main steps: formation of silanol (Si-OH) groups on the surface of BG, silica dissolution, and the development of an amorphous calcium phosphate layer, which in turn crystallizes as HAP [8]. As both human bone and dentin are similar in their chemical compositions, it can be predicted that the material, which forms a strong bond with the former, will also form a similar bond with the latter [24]. Bakri et al. [25], based on their in vitro analysis of dentin tubule occlusion competence of a BG dentifrice and comparison of that result with that of an arginine and fluoride containing dentifrice, conclude that after acidic challenge, BG toothpaste shows better tubule occlusion than other groups. Previously, Shaikh et al. [11], report that BioMinF ${ }^{\mathbb{C}}$ demonstrates better blockage of patent tubules of dentin discs as compared to Novamin ${ }^{\circledR}$ dentifrice. In the present study, we observed similar tubule occlusion competence for BioMinF $^{\odot}$. Our novel BG-containing dentifrices also showed similar results in respect of $\mathrm{BioMinF}^{\odot}$. The presence of BG (1.5 and 4\% wt/wt.) BG in those dentifrices could have contributed to better tubule occlusion by virtue of its surface reaction with dentin, which ensures enhanced plugging of open tubules. Moreover, nano- $\mathrm{ZnO}$ powders in experimental dentifrices offer an additional advantage. $\mathrm{ZnO}$ powder ( $3 \% \mathrm{wt}$.) is added as a preservative to toothpastes, which in aqueous suspension inhibits not only dentin demineralization but also induces antimicrobial action by yielding zinc ions $\left(\mathrm{Zn}^{2+}\right)$ and reactive oxygen species [25]. The partial dissolution of $\mathrm{ZnO}$ particles releases $\mathrm{Zn}^{2+}$ ions in aqueous suspension, which display antimicrobial action against many bacterial and fungal strains through direct contact with cell wall [26].

The ideal dentifrice for treating $\mathrm{DH}$ should not only block the tubules but also preserve the occlusion of tubules when faced with acidic challenge [10]. The experimental dentifrices of this study blocked a majority of the tubules and also retained most of the tubule occlusion,
Table 3. Statistical values when pre- versus post-citric acid challenge tubule occlusion values were compared within each group

\begin{tabular}{|c|c|c|c|c|c|c|c|c|}
\hline \multicolumn{3}{|c|}{ Groups } & \multicolumn{3}{|c|}{$\begin{array}{l}\text { Paired samples test, } \\
\text { paired differences }\end{array}$} & \multirow[t]{2}{*}{$t$} & \multirow[t]{2}{*}{$\mathrm{df}$} & \multirow[t]{2}{*}{$\begin{array}{l}p \\
\text { value }\end{array}$} \\
\hline & & & mean & $\mathrm{SD}$ & SEM & & & \\
\hline 2 & & & & 0.75 & & & 7 & 0.001 \\
\hline 3 & Pair 1 & Pre - post & 2.00 & 0.92 & & & 7 & 0.000 \\
\hline 4 & Pair 1 & Pre - post & 2.50 & 1.51 & & & 7 & 0.002 \\
\hline 5 & Pair 1 & Pre - post & 2.87 & 3.09 & 1.09279 & 2.631 & 7 & 0.034 \\
\hline 6 & Pair 1 & Pre - post & 2.62 & 1.76 & 0.62500 & 4.200 & 7 & 0.004 \\
\hline
\end{tabular}

Paired sample $t$ test, group 1 values are not shown as they resulted in no tubule occlusion.

Table 4. Showing Tukey HSD - post hoc test results when postcitric acid challenge tubule occlusion values were compared among the groups

\begin{tabular}{lllll}
\hline Group & Group(s) & Mean difference & SE & $p$ value \\
\hline 2 & 3 & 1.12 & 2.36 & 0.999 \\
& 4 & $-60.37^{*}$ & 2.36 & 0.000 \\
& 5 & $-67.12^{*}$ & 2.36 & 0.000 \\
& 6 & $-64.50^{*}$ & 2.36 & 0.000 \\
\hline 3 & 4 & $-61.50^{*}$ & 2.36 & 0.000 \\
& 5 & $-68.25^{*}$ & 2.36 & 0.000 \\
& 6 & $-65.62^{*}$ & 2.36 & 0.000 \\
\hline 4 & 5 & -6.75 & 2.36 & 0.084 \\
& 6 & -4.12 & 2.36 & 0.588 \\
\hline
\end{tabular}

* The mean difference is significant at the 0.05 level.

post-acidic challenge. The traditional BG composition $\left(45 \mathrm{~S} 5\right.$, Bioglass $\left.^{\odot}\right)$ is devoid of fluoride and contains only calcium sodium phosphosilicate [27]. The dentifrice, based on Novamin ${ }^{\odot}$, is one such example as it contains BG, but no fluoride, in its glass composition; so, soluble fluoride needs to be added in the formation of the dentifrice [10]. To achieve maximum benefits of fluoride, it should be released and deposited slowly on to the surface of the tooth [10]. The presence of fluoride inside the BG composition ensures slow and sustained release of fluoride with the possibility of forming long-lasting FAP, rather than fluorite [6]. Previously, Farooq et al. [27] synthesized new fluoride-containing BG compositions, which formed FAP in Tris buffer solution at a faster rate than 45S5. Another study on the effect of adding fluoride 
to BG composition showed that fluoride softens the glass, and is thus more bioactive [28]. Therefore, the superiority of the two experimental BG dentifrices in tubule occlusion potential can be attributed to the presence of fluoride in the BG structure.

Zinc, though present only in small amounts in the human body, plays an important role in cell division, growth, and bone metabolism [29]. Considering all these benefits, zinc was included in the experimental BG-containing dentifrices, the effect of which is evident in the results of Energy-dispersive $\mathrm{X}$-ray analysis. The in vitro nature of this study could be considered a limitation to obtaining fully reliable results, as the in vivo conditions could be quite different from the in vitro conditions. Another limitation could be the manual counting of blocked tubules, which could be false due to human error. However, every attempt was made to minimize the counting error by standardizing all the variables. Well-controlled clinical trials need to be conducted to further analyze the effect of these experimental dentifrices in actual dynamic in vivo conditions. The results of this study should be considered only as tentative findings, which need to be followed up by long-term assessment of the tested dentifrices.

\section{Conclusion}

We conclude that dentifrices containing fluoridezinc-BG have better tubule occlusion potential compared to other dentifrices. The results of these experimental dentifrices are comparable to those of BioMinF $^{\odot}$ and are in conformity with the prevailing opinion in the literature that BGs containing fluoride and zinc occlude the tubules efficiently, and hence can be used for the treatment of $\mathrm{DH}$.

\section{Acknowledgments}

The authors are grateful to Dr. Asma Tufail Shah and Dr. Aqif Anwar Chaudhry, Interdisciplinary Research Centre in Biomedical Materials, COMSATS University, Islamabad, Lahore Campus, Lahore, Pakistan, for providing fluoridated-BG and $\mathrm{ZnO}$. The authors are also thankful to Mr. Intisar Siddiqui for providing statistical support.

\section{Disclosure Statement}

The authors have no conflicts of interest to declare.

\section{References}

1 Farooq I, Moheet IA, AlShwaimi E. In vitro dentin tubule occlusion and remineralization competence of various toothpastes. Arch Oral Biol. 2015 Sep;60(9):1246-53.

2 Brännström M. Sensitivity of dentine. Oral Surg Oral Med Oral Pathol. 1966 Apr;21(4): 517-26.

3 Najeeb S, Khurshid Z, Zafar MS, Ajlal S. Applications of light amplification by stimulated emission of radiation (lasers) for restorative dentistry. Med Princ Pract. 2016;25(3):20111.

4 Vasiliadis L, Darling AI, Levers BG. The histology of sclerotic human root dentine. Arch Oral Biol. 1983;28(8):693-700.

5 Kara C, Orbak R. Comparative evaluation of $\mathrm{Nd}$ :YAG laser and fluoride varnish for the treatment of dentinal hypersensitivity. J Endod. 2009 Jul;35(7):971-4.

6 Farooq I, Imran Z, Farooq U, Leghari MA, Ali H. Bioactive glass: A material for the future. World J Dent. 2012;3(2):199-201.

7 Gjorgievska E, Nicholson JW. Prevention of enamel demineralization after tooth bleaching by bioactive glass incorporated into toothpaste. Aust Dent J. 2011 Jun;56(2):193-200.

8 Khalid MD, Khurshid Z, Zafar MS, Farooq I, Khan RS, Najmi A. Bioactive glasses and their applications in dentistry. J Pak Dent Assoc. 2017;26(1):32-8.
9 Lucas PW, van Casteren A. The wear and tear of teeth. Med Princ Pract. 2015;24(Suppl 1): 3-13.

10 Alhussain AM, Alhaddad AA, Ghazwi MM, Farooq I. Remineralization of artificial carious lesions using a novel fluoride incorporated bioactive glass dentifrice. Dent Med Probl. 2018 Oct-Dec;55(4):379-82.

11 Shaikh K, Pereira R, Gillam DG, Phad S. Comparative Evaluation of Desensitizing Dentifrices containing BioMin ${ }^{\circledR}$, Novamin ${ }^{\circledR}$ and Fluoride on Dentinal Tubule Occlusion before and after a Citric Acid Challenge - A scanning Electron Microscope in-vitro Study. J Odontol. 2018;2:105.

12 Kanwal N, Brauer DS, Earl J, Wilson RM, Karpukhina N, Hill RG. In-vitro apatite formation capacity of a bioactive glass - containing toothpaste. J Dent. 2018 Jan;68:51-8.

13 Cummins D. The superior anti-caries efficacy of fluoride toothpaste containing 1.5\% arginine. J Clin Dent. 2016 Jun;27(2):27-38.

14 Ahtzaz S, Nasir M, Shahzadi L, Amir W, Anjum A, Arshad R, et al. A study of the effect of zinc oxide and zinc peroxide nanoparticles to enhance angiogenesis-pro-angiogenic grafts for tissue regeneration applications. Mater Des. 2017;132:409-18.
15 Gul H, Zahid S, Zahid S, Kaleem M, Khan AS, Shah AT. Sol-gel derived fluoride-doped bioactive glass powders: structural and longterm fluoride release/pH analysis. J NonCryst Solids. 2018;498:216-22.

16 Fusayama T, Katayori T, Nomoto S. Corrosion of gold and amalgam placed in contact with each other. J Dent Res. 1963 Sep-Oct; 42(5):1183-97.

17 Pathan AB, Bolla N, Kavuri SR, Sunil CR, Damaraju B, Pattan SK. Ability of three desensitizing agents in dentinal tubule obliteration and durability: an in vitro study. J Conserv Dent. 2016 Jan-Feb;19(1):31-6.

18 Mäkinen KK. Sugar alcohol sweeteners as alternatives to sugar with special consideration of xylitol. Med Princ Pract. 2011;20(4):30320.

19 Memarpour M, Fakhraei E, Dadaein S, Vossoughi M. Efficacy of fluoride varnish and casein phosphopeptide-amorphous calcium phosphate for remineralization of primary teeth: a randomized clinical trial. Med Princ Pract. 2015;24(3):231-7.

20 da Cruz LP, Hill RG, Chen X, Gillam DG. Dentine tubule occlusion by novel bioactive glass-based toothpastes. Int J Dent. 2018 Apr; 2018:5701638. 
21 Aasenden R, Brudevold F, Richardson B. Clearance of fluoride from the mouth after topical treatment or the use of a fluoride mouthrinse. Arch Oral Biol. 1968 Jun;13(6): 625-36.

$22 \mathrm{Ma}$ J, Johns RA, Stafford RS. Americans are not meeting current calcium recommendations. Am J Clin Nutr. 2007 May;85(5):13616.

23 Lynch E, Brauer DS, Karpukhina N, Gillam DG, Hill RG. Multi-component bioactive glasses of varying fluoride content for treating dentin hypersensitivity. Dent Mater. 2012 Feb;28(2):168-78.
24 Kanel SR, Al-Abed SR. Influence of $\mathrm{pH}$ on the transport of nanoscale zinc oxide in saturated porous media. J Nanopart Res. 2011;13(9): 4035-47.

25 Bakri MM, Hossain MZ, Razak FA, Saqina $\mathrm{ZH}$, Misroni AA, Ab-Murat N, et al. Dentinal tubules occluded by bioactive glass-containing toothpaste exhibit high resistance toward acidic soft drink challenge. Aust Dent J. 2017 Jun;62(2):186-91.

26 Pasquet J, Chevalier Y, Pelletier J, Couval E, Bouvier D, Bolzinger MA. The contribution of zinc ions to the antimicrobial activity of zinc oxide. Colloids Surf A Physicochem Eng Asp. 2014;457:263-74.
27 Farooq I, Tylkowski M, Müller S, Janicki T, Brauer DS, Hill RG. Influence of sodium content on the properties of bioactive glasses for use in air abrasion. Biomed Mater. 2013 Dec; 8(6):065008.

28 Brauer D, Karpukhina N, Law R, Hill R. Structure of fluoride containing bioactive glasses. J Mater Chem. 2009;19(31):5629-36.

29 Ali S, Farooq I, Iqbal K. A review of the effect of various ions on the properties and the clinical applications of novel bioactive glasses in medicine and dentistry. Saudi Dent J. 2014 Jan;26(1):1-5. 